\title{
Lanreotide depot deep subcutaneous injection: a new method of delivery and its associated benefits
}

This article was published in the following Dove Press journal:

Patient Preference and Adherence

17 January 2012

Number of times this article has been viewed

\section{John D Carmichael}

Department of Medicine, Cedars-Sinai Medical Center, Los Angeles, CA, USA
Correspondence: John D Carmichael 8700 Beverly Boulevard, Los Angeles, CA 90048, USA

$\mathrm{Tel}+\mathrm{I} 3104232830$

Fax +13104232819

Email carmichaelj@cshs.org

\begin{abstract}
Acromegaly is a rare disease characterized by excessive growth hormone secretion, usually from a pituitary tumor. Treatment options include surgery, medical therapy, and in some cases, radiation therapy. Current medical therapy consists of treatment with somatostatin analog medications or a growth hormone receptor antagonist. There are two somatostatin analogs currently in use, octreotide and lanreotide. Both are supplied in long-acting formulations and are of comparable biochemical efficacy. Lanreotide is supplied in a prefilled syringe and is injected into deep subcutaneous tissue. Studies have been conducted to assess the efficacy of self- or partner administration, and have demonstrated that injection of lanreotide can be accomplished reliably and safely outside a physician's office. For patients who have achieved biochemical control with lanreotide, the FDA has recently approved an extended dosing interval. Selected patients may be able to receive the medication less frequently with injections of $120 \mathrm{mg}$ administered every 6 or 8 weeks. This review focuses on the use of lanreotide in the treatment of acromegaly, the safety and efficacy of the drug, and the benefits afforded to patients because of unique aspects of the delivery of lanreotide.
\end{abstract}

Keywords: acromegaly, treatment, lanreotide, somatostatin analog, pituitary tumor

\section{Introduction}

Acromegaly is a chronic, insidious disease caused by excess growth hormone $(\mathrm{GH})$ secretion, usually from a pituitary adenoma. Uncontrolled disease is associated with an approximately $2-4$ fold increased mortality ${ }^{1,2}$ and multiple comorbidities. ${ }^{3}$ Treatment of acromegaly consists of a combination of modalities including surgery, medical therapy, and radiation therapy. ${ }^{4,5}$ Medical therapy, either for adjuvant therapy or primary medical therapy, consists of somatostatin analogs or GH receptor antagonists. Somatostatin analogs act to decrease GH secretion from the pituitary adenoma or remnant tissue, thereby reducing insulin-like growth factor one (IGF-I) levels, and reducing signs and symptoms associated with acromegaly.

Many patients undergo surgery as their primary therapy; however, patients who present with invasive tumors require medical therapy after non-curative surgery. Estimated rates of surgical cure with an experienced surgeon depend on tumor size, and approximately $40 \%$ of all patients require some medication after primary surgery. ${ }^{6}$ Somatostatin analogs induce biochemical control in approximately $50 \%-60 \%$ of treated patients. ${ }^{7}$ The two drugs available in this class are long-acting injectable medications, octreotide (Sandostatin LAR, Novartis, Basel, Switzerland) and lanreotide (Somatuline Depot, Ipsen, Paris, France). Both medications are of comparable efficacy and have similar molecular structure and mechanisms of action. ${ }^{4}$ One key difference between these medications is 
how the drugs are supplied and how this difference impacts the administration of the drugs. Another important difference is the recent FDA approval of an extended dosing interval between injections in patients controlled with lanreotide, making it possible to administer this medication less frequently, thereby reducing the cost of care for these patients.

This review focuses on the delivery system utilized by lanreotide and the benefits that this delivery system provides. Prescribing information is discussed and elements of the delivery of this drug that provide benefit to patients and prescribers will be reviewed. This paper critically reviews the impact lanreotide has on treatment of acromegaly, the effect on quality of life, and the level of satisfaction garnered by patients using the medication.

\section{Background}

Acromegaly is a rare disease, with an incidence approximated at 3-4 cases per million per year, ${ }^{8}$ and estimated prevalence of 8.6 per 100,000 or higher. ${ }^{9,10}$ The onset is insidious with diagnosis often delayed for many years. ${ }^{11,12}$ Left untreated, the disease causes a significant increase in mortality, due primarily to cardiovascular and cerebrovascular disease. ${ }^{1,2}$ Treatment of acromegaly improves the increased risk of mortality, decreases comorbidity, and improves quality of life. Reduction in serum GH levels and normalization of serum IGF-I levels are associated with a return of mortality risk to that of a control population. ${ }^{13,14}$

The goals of therapy include control of GH secretion, control of the tumor mass, reduction in associated comorbidities and symptoms of acromegaly, and preservation of normal pituitary function. Treatment modalities currently employed include surgery, medical therapy, and radiation therapy. Recent advances have furthered the field of acromegaly treatment, including improvements in surgical techniques incorporating endoscopic instruments and techniques employed for stereotactic radiation, as well as novel medications and medication delivery systems.

Surgical resection of a GH secreting pituitary tumor is the primary treatment of choice for most of the patients with acromegaly. ${ }^{15}$ In experienced hands, the surgical cure rate is high for microadenomas (maximum diameter $<10 \mathrm{~mm}$ ), but the cure rate declines when tumors are invasive or for macroadenomas (diameter $>10 \mathrm{~mm}$ ). ${ }^{6}$ When surgery is successful, as measured by normalization of elevated IGF-I, and suppression of GH by a glucose load, patients continue to require monitoring for tumor recurrence but do not need further treatment of excess growth hormone. If surgery is not curative, further treatment is required.
There are multiple medications available to treat acromegaly. Dopamine agonist medications, bromocriptine and cabergoline, have been used to treat acromegaly and work by suppressing GH secretion from the somatotroph adenoma. Somatostatin analogs suppress GH secretion and have been shown to have effects on tumor shrinkage. The GH receptor antagonist pegvisomant is the only drug in its class, ${ }^{16}$ and effectively blocks the effects of $\mathrm{GH}$ at the level of the $\mathrm{GH}$ receptor, reducing IGF-I levels and controlling symptoms. ${ }^{17}$ Use of these medications in combination has also been published, with increased efficacy shown with combination therapy of dopamine agonists and somatostatin analogs, ${ }^{18}$ as with combined use of pegvisomant and somatostatin analogs. ${ }^{19,20}$

Treatment with somatostatin analogs is generally well tolerated, efficacious in approximately $60 \%$ of patients, and has effects on both biochemical parameters (GH and IGF-I secretion) as well as effects on tumor mass. ${ }^{21}$ Somatostatin analogs may be used as adjuvant therapy after pituitary surgery or as primary therapy in selected patients. ${ }^{15}$ Somatostatin analog medications have been shown to have positive effects on symptoms, GH secretion, serum IGF-I levels, tumor size, comorbidities and effects of chronic GH secretion. However, many patients treated with somatostatin analogs will not normalize their GH and IGF-I levels, and require further treatment.

In addition to medical therapy, radiation therapy including conventional radiation, stereotactic conventional radiation therapy, gamma knife therapy, proton beam - has been used to control tumor mass and reduce GH secretion. ${ }^{22}$ Use of radiation in the treatment of patients with acromegaly has been associated with an increased rate of mortality and cerebrovascular events. ${ }^{23,24}$ Despite the adverse effects that limit its appeal, radiation therapy remains an integral part of treatment for challenging patients with acromegaly. Its use varies in different treatment centers, with some reserving its use for only the most difficult cases, while others use the treatment more frequently in lieu of costly, lifelong medical treatment.

Monitoring patients with acromegaly usually entails regular assessments of serum GH and IGF-I levels, MRI surveillance, and careful regular assessments and treatments of comorbidities associated with acromegaly. Chronic GH excess can have profound effects on metabolism, the cardiovascular system, and joints, making regular assessment of glucose metabolism, cardiovascular disease, and arthritis a lifelong component of acromegaly care. Furthermore, effects on sleep apnea and the increased 
risk of developing colon polyps requires assessment for ongoing sleep apnea and regular colonoscopy even when biochemical control or cure is attained. Safety monitoring of medical therapy requires MRI surveillance and assessment of transaminase levels for pegvisomant, but no regular safety surveillance is required specifically for somatostatin analogs. Physicians should be aware of potential side effects of cholecystitis or gallstone formation, however, and assess complaints consistent with these side effects with gall bladder ultrasound.

\section{Drug profile Mechanism of action}

Lanreotide is a synthetic octapeptide that is an analog of the biologically active portion of somatostatin, a 14-amino acid peptide that binds to somatostatin receptors (SSTR). There are at least five SSTR subtypes that are g-protein coupled receptors with seven trans-membrane spanning domains. ${ }^{25}$ Lanreotide has a high affinity to SSTR subtype 2 (SSTR2) and SSTR subtype 5 (SSTR5). These receptor subtypes regulate $\mathrm{GH}$ secretion from pituitary somatotroph cells and their activity is retained in most pituitary adenomas secreting $\mathrm{GH}$. Binding of lanreotide to the SSTR2 and SSTR5 results in decreased GH secretion from the somatotroph tumor. ${ }^{25}$

Since lanreotide is available in multiple formulations worldwide, and the medical literature is replete with multiple studies investigating the safety, efficacy, and use of these formulations, reviewing the use of this medication can be complex. Lanreotide was initially supplied as a prolonged release medication that utilized a microparticle vehicle to sustain the actions of lanreotide once administered. This formulation is known as the prolonged release (PR) or sustained release (SR) formulation. The PR formulation is administered every 7-14 days in a fixed dose and demonstrates good efficacy and tolerability in treatment of acromegaly. ${ }^{26}$ Later, lanreotide became available in a preparation that delivered more sustained levels of drug through a depot formulation. This formulation is a supersaturated gel of lanreotide and water only, without other incipients. This formulation is in current use in the US and worldwide. Outside the US, it is known as the autogel formulation, and in the US it is known as Somatuline depot formulation. Thus, many studies conducted prior to the drug's release in the US or outside the US describe this medication as "lanreotide autogel," while studies conducted after its FDA approval in the US may refer to it as "lanreotide depot formulation" (Table 1). ${ }^{35-47}$

\section{Pharmacodynamics}

Much of the knowledge regarding the pharmacokinetics and pharmacodynamics of the depot formulation is derived from clinical experiments using lanreotide in the PR formulation and the autogel formulation, as well as pharmacokinetic data derived from various computer models. ${ }^{27}$

Similar to native somatostatin, lanreotide exhibits endocrine effects throughout the body through coupling with SSTRs. ${ }^{25}$ The primary pharmacodynamics effect is the reduction of $\mathrm{GH}$ secretion, resulting in decreased production of IGF-I in various tissues. Somatotroph pituitary adenomas predominately express SSTR2 and 5, and increased affinity for these subtypes by synthetic somatostatin receptor ligands such as lanreotide provide for the primary pharmacodynamics effect of reduction in $\mathrm{GH}$ secretion. SSTRs are also expressed throughout the gastrointestinal tract and pancreas, accounting for the known adverse effects related to gastrointestinal motility. ${ }^{28}$ Lanreotide inhibits basal motilin, gastric inhibitory peptide, and pancreatic polypeptide secretion. Postprandial secretion of pancreatic polypeptide, gastrin, and cholecystokinin are inhibited by lanreotide, and in healthy subjects postprandial insulin secretion is delayed, resulting in transient mild glucose intolerance. ${ }^{29}$ Other non-pituitary effects observed include increased amylase secretion in healthy individuals, and delayed postprandial bicarbonate secretion in the duodenum, resulting in reduction in gastric acidity. ${ }^{29}$

\section{Pharmacokinetics}

The pharmacokinetic profile of lanreotide depot has been evaluated in healthy subjects and patients with acromegaly. ${ }^{27,30,31}$ Half-life, as determined by injection in healthy volunteers after a single administration, was 21-22 days. ${ }^{30}$ During administration of fixed doses every 28 days over a 4 month clinical trial, pharmacokinetics were established after a single dose and at steady state. ${ }^{31}$ The depot formulation showed linear pharmacokinetic profiles in three different dosages $(60,90$, and $120 \mathrm{mg})$ after a single injection and repeated administration (Figure 1). There was no apparent burst effect after administration and a stable linear release profile was seen over the following 28 days. Minimum and maximum concentrations and area under the curve followed expected dose proportionality. Based on population pharmacokinetics, and software-assisted modeling of drug disposition, minimum effective levels of lanreotide were found with a dose of $120 \mathrm{mg} 56$ days after injection. ${ }^{27,32}$ With this information, the possibility of extending the interval between injections was suggested. Extended dosing intervals have recently been FDA approved. 


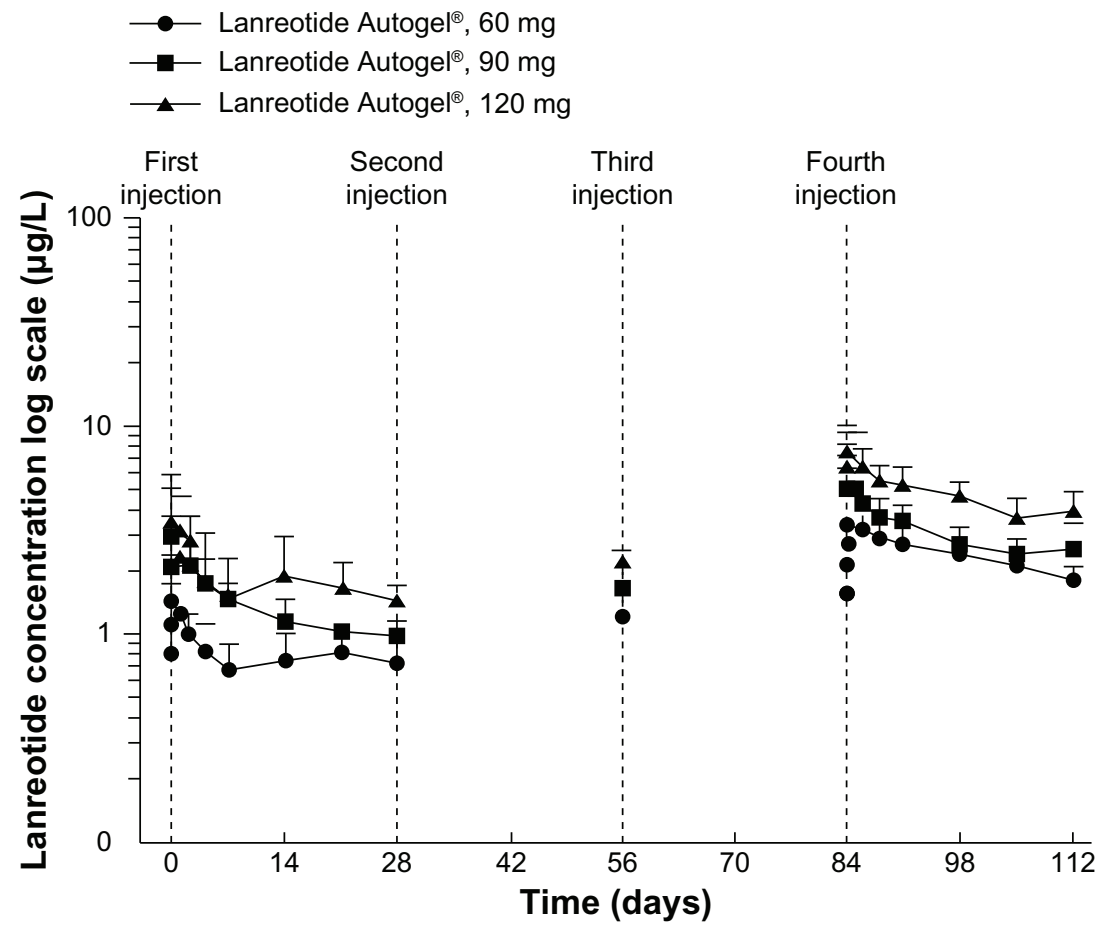

Figure I Comparative (mean \pm standard deviation) serum level profiles of lanreotide following one and four injections of lanreotide Autoge ${ }^{\circledR}$ at doses of 60,90 , and 120 mg. From Bronstein M, Musolino N, Jallad R, et al. Pharmacokinetic profile of lanreotide Autogel in patients with acromegaly after four deep subcutaneous injections of 60 , 90 , or $120 \mathrm{mg}$ every 28 days. Clin Endocrinol. 2005;63(5):514-519. With permission.

\section{Therapeutic efficacy}

Therapeutic efficacy for lanreotide in the PR formulation has been well established, and since the advent of the autogel or depot formulation, the efficacy of the newer formulation has been increasingly demonstrated in multiple clinical trials. The following review will focus only on trials involving the currently available depot or autogel formulation, used alone in the treatment of acromegaly. Clinical trials where efficacy of the drug was the primary endpoint will be reviewed.

To date, while there have been several small studies conducted observing clinical endpoints in treatment of acromegaly with various forms of lanreotide and octreotide, no study has been large enough or adequately powered to demonstrate superiority of one drug over another. The efficacy rates, in terms of biochemical endpoints, of all somatostatin analog treatments vary among multiple clinical trials. For adjuvant therapy, GH and IGF-I control ranges from approximately $30 \%-75 \%$, whereas primary medical therapy for $\mathrm{GH}$ and IGF-I control ranges from approximately $40 \%-75 \% .{ }^{33}$

Two systematic reviews of the use of somatostatin analogs, including lanreotide, have been published. ${ }^{26,34}$ The first evaluated 44 trials of octreotide LAR and lanreotide PR, and did not include data on the autogel formulation. ${ }^{26}$ The second critical review of somatostatin analog use included large studies using octreotide, octreotide LAR, lanreotide
PR, and lanreotide autogel/depot formulation. ${ }^{34}$ This analysis provides a substantial overview of the use of these medications in the treatment of acromegaly, and suggests that the two long-acting preparations, octreotide LAR and lanreotide autogel, are comparable in their efficacy. Establishing any superiority of one medication over another would require a randomized, controlled trial.

Table 1 summarizes the biochemical efficacy endpoints for several studies evaluating the use of lanreotide autogel/ depot in the treatment of acromegaly. ${ }^{35-47}$ Trials listed in the table specifically investigated biochemical outcomes and published rates of GH and IGF-I control. Additional trials have been reported examining effects of lanreotide on outcomes such as tumor size, cardiovascular effects, and glucose metabolism. ${ }^{40,48,49}$ Most of the clinical trials investigating the efficacy of lanreotide have been conducted on subjects who received treatment of one form or another (surgical, radiation, or medical) prior to study entry. Specifically, many studies evaluated the efficacy of lanreotide after transitioning from either lanreotide as supplied in the PR formulation or from octreotide LAR. Hence, these studies, and the efficacy rates they quote, need to be evaluated with the knowledge that there may be a selection bias in the response rates as published. Similarly, few studies have been conducted with randomization or stratification. 


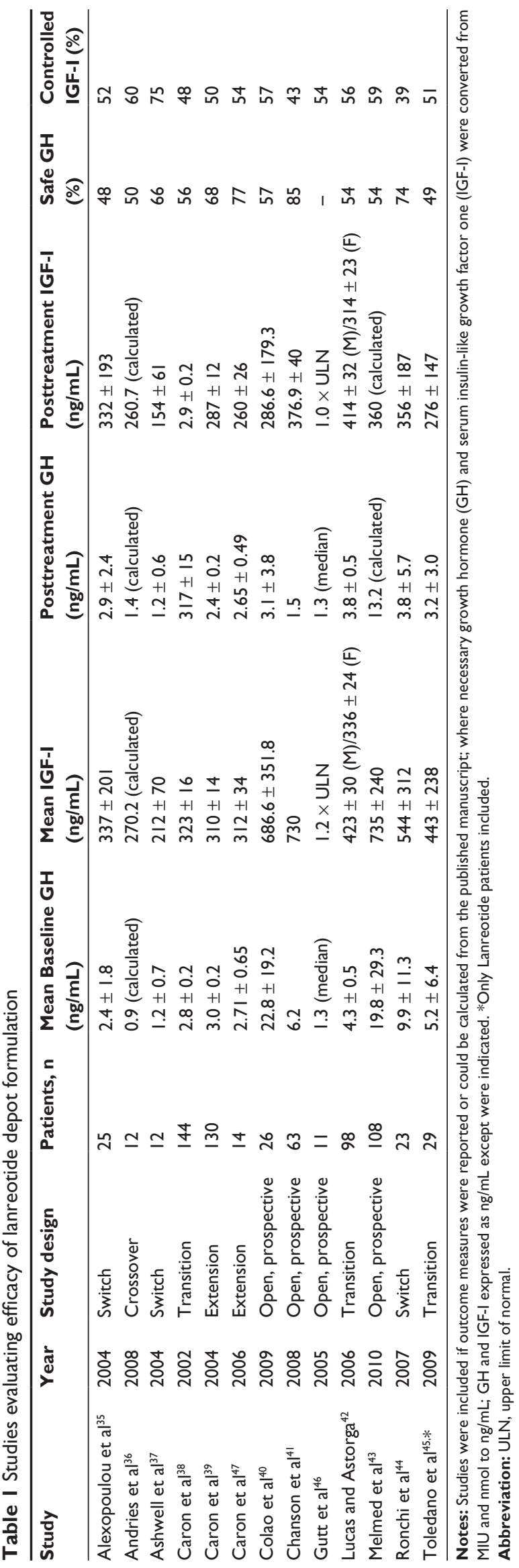

Based on published studies, dose titration does appear to improve the efficacy rates in patients treated with lanreotide. In addition, the majority of patients who have participated in these studies required escalation of the dose up to the maximum of $120 \mathrm{mg}$ every 28 days. A study evaluating alternative dosing schemes investigated whether decreasing the interval for subjects requiring a dose higher than $120 \mathrm{mg}$ every 28 days found no further improvement with a dose of $120 \mathrm{mg}$ every 21 days. $^{50}$

\section{Tolerability/Safety}

The safety and tolerability of lanreotide has been addressed by multiple studies as well as post marketing surveillance. ${ }^{29}$ The drug is well tolerated and has been used for many years in treatment of acromegaly. Pooled adverse events reported at $>5 \%$ in 416 patients from seven clinical trials are presented in Table 2. The most common side effects are gastrointestinal complaints including diarrhea, abdominal pain, loose stools, flatulence, nausea, constipation, and vomiting. In a placebocontrolled trial comparing different doses of lanreotide, the gastrointestinal symptoms were reported in higher frequency with higher doses.$^{29}$ Cholelithiasis and injection-site reactions are also commonly observed. Gastrointestinal complaints occur more severely and frequently when the medication is initiated, and become less bothersome over the course of treatment. Cholelithiasis is observed in subjects receiving somatostatin analogs, and has been observed as biliary sludge and asymptomatic stones more frequently than symptomatic biliary obstruction. Injection site reactions, including injection site pain, mass, and induration were reported at a higher frequency at the beginning of therapy, and decreased in incidence as treatment progressed. ${ }^{29}$

Changes in blood glucose levels (hyperglycemia, hypoglycemia, or diabetes) were reported in $14 \%$ of subjects in seven clinical trials with $7 \%$ related to the study drug. ${ }^{29}$ Somatostatin analogs are known to delay insulin secretion after a glucose load, which may alter blood glucose metabolism and have a causative role in altered glucose metabolism. Sinus bradycardia was the most commonly reported cardiac adverse event, but associations with the study drug could not be clearly established due to the high prevalence of cardiac disease in these subjects. ${ }^{29}$

\section{Patient adherence and satisfaction Self- or partner injection}

One of the key elements to patient satisfaction is ease of administration and access to medication. The advent of an injectable medication in a prefilled syringe has improved 
Table 2 Adverse reactions at an incidence $>5 \%$ reported in selected clinical studies

\begin{tabular}{|c|c|c|c|c|}
\hline \multirow[t]{3}{*}{ System organ class } & \multicolumn{4}{|c|}{ Number and percentage of patients } \\
\hline & \multicolumn{2}{|c|}{$\begin{array}{l}\text { Pivotal studies } I \text { and } 2 \\
(\mathrm{~N}=\mid 70)\end{array}$} & \multicolumn{2}{|c|}{$\begin{array}{l}\text { Overall pooled data } \\
(N=416)\end{array}$} \\
\hline & $\overline{\mathbf{N}}$ & $\%$ & $\overline{\mathbf{N}}$ & $\%$ \\
\hline Patients with any adverse reactions & 157 & 92 & 356 & 86 \\
\hline Gastrointestinal disorders & $|2|$ & 71 & 235 & 57 \\
\hline Diarrhea & 81 & 48 & 37 & 155 \\
\hline Abdominal pain & 34 & 20 & 19 & 79 \\
\hline Nausea & 15 & 9 & 11 & 46 \\
\hline Constipation & 9 & 5 & 8 & 33 \\
\hline Flatulence & 12 & 7 & 7 & 30 \\
\hline Vomiting & 8 & 5 & 7 & 28 \\
\hline Loose stools & 16 & 9 & 6 & 23 \\
\hline Hepatobiliary disorders & 53 & 31 & 99 & 24 \\
\hline Cholelithiasis & 17 & 45 & 20 & 85 \\
\hline General disorders and administration site conditions & 51 & 30 & 91 & 22 \\
\hline (Injection site pain/mass/induration/nodule/pruritus) & 17 & 28 & 37 & 9 \\
\hline Musculoskeletal and connective tissue disorders & 44 & 26 & 70 & 17 \\
\hline Arthralgia & 17 & 10 & 30 & 7 \\
\hline Nervous system disorders & 34 & 20 & 80 & 19 \\
\hline Headache & 9 & 5 & 30 & 7 \\
\hline \multicolumn{5}{|l|}{ Cardiac system disorders } \\
\hline Sinus bradycardia & 12 & 7 & 13 & 3 \\
\hline Hypertension & 11 & 7 & 20 & 5 \\
\hline Anemia & 12 & 7 & 14 & 3 \\
\hline
\end{tabular}

Note: Information from Somatuline Depot Prescribing information. ${ }^{29}$

access to medication, especially for those who need to travel to obtain monthly injections and find this is a hardship. Lanreotide, since it does not require reconstitution, can be injected by a trained individual (either the patient or a trusted "partner") without concern of variability in drug preparation. Self-administration has been widely approved in European countries and since there is no statement requiring administration of the drug by a medical professional in the prescribing information, the medication can be administered outside the confines of the medical office or hospital.

Two multicenter studies have been conducted to evaluate the safety and efficacy of self- or partner injection, one in the UK and another in the US..$^{51,52}$ In the UK study, 30 subjects were recruited to assess the ability of patients or their partners to safely and reliably administer multiple doses of the medication in an unsupervised setting. ${ }^{52}$ The primary endpoint was the proportion of subjects who were able to successfully administer the medication throughout the study. Multiple centers throughout the UK were involved in the clinical trial, which was an open-label controlled study of 36 months. In this study, patients self-selected at the screening visit whether to receive lanreotide as administered by a health care professional (control subjects) or by either themself or a partner (test subjects). Doses were not titrated during the study and stable doses (over at least 4 months) were required prior to study entry. Test subjects were trained to inject the lanreotide at the baseline visit and performed occasional supervised injections for training as needed. Once trained, the individuals performed home injections, unsupervised, for the duration of the trial. There were 15 subjects in the test group and 15 in the control group. Subjects in the test group were younger and more likely to have undergone pituitary surgery. Prior treatment with somatostatin analogs was longer in duration for the control group. All test subjects were quickly deemed suitable for home injection (by partner or self) after one training session. Nearly all subjects in the test group (93\%) were able to successfully administer the injection at home. The one subject who did not meet the endpoint criteria had an increase in mean GH to $>5 \mathrm{ng} / \mathrm{mL}$ at study end. GH and IGF-I control was maintained during the trial, however, as assessed at the interim visit. Two subjects (one in the test group and one in the control group) did not demonstrate controlled biochemistry at study end. No subjects withdrew from the study. Pain associated with the injection was not reported any worse in the partner or self-administered group.

The US study differed in its methods but also explored the ability of subjects to self- or partner administer lanreotide. ${ }^{51}$ 
This was a 6-month open-label single-arm study, conducted at multiple centers in the US. While the UK study required stable dosing of lanreotide 4 months prior to entry, the US study enrolled subjects who opted to switch from prior therapy with Sandostatin LAR (switch patients) or were either drug naïve (naïve patients) or not currently receiving treatment (no medication for 4 months prior to entry). These patients were collectively described as "other" patients. Dosing of the lanreotide at study entry depended upon the dose of prior therapy (if any) or was set at $90 \mathrm{mg}$ monthly for naïve subjects. Dosing was adjusted 16 weeks after study entry based on serum IGF-I levels and symptoms.

At the baseline visit, patients were given the option of self-administration or partner administration. Subjects and partners were trained, and study staff utilized a questionnaire to assess competency on a monthly basis during the first 3 months of the study. A diary and a patient questionnaire assessed the patients' symptoms throughout the study period. As with the UK study, the primary endpoint was the percentage of patients or partners who were able to successfully administer lanreotide without supervision of a health care professional. Fifty-nine patients were recruited and seven of these were withdrawn from the clinical trial. Seventy percent of subjects in the trial preferred to self-inject. All subjects were assessed as being competent to self- or partner administer their medications by week 4 and throughout the study. A significant number of switch patients found their injections to be less painful than those of Sandostatin LAR (50\% vs $25 \%$ ) and switch patients found the lanreotide injection very convenient compared to Sandostatin LAR (75\% vs $18 \%$; $P=0.0002)$. Most patients who switched to lanreotide for the trial reported that they preferred lanreotide over Octreotide LAR for future use ( $81 \%$ vs $12 \% ; P=0.0001)$

Control, measured by serum IGF-I level, was achieved in the majority of switch patients at the study end. At baseline, $84 \%$ of switch patients entered the study with controlled IGF-I, and 94\% achieved normal IGF-I values at study end. Forty-three percent of other patients achieved a normal IGF-I at study end. Symptoms were either improved or similar in switch and other patients.

The results of these studies indicate that patients are able and willing to self-inject or have a partner assist with injections outside a medical office. The studies confirm that the use of the prefilled syringe, which is ready for injection without the requirement drug reconstitution, offers convenience without sacrificing disease control. The use of lanreotide by a non-health care professional is aided by the fact that the medication need not be injected into the muscle but is intended for deep subcutaneous injection. This allows for a short needle, thus making the home injection process less intimidating than an intramuscular injection.

\section{Quality of life and symptom control}

Quality of life and symptom control have only been reported briefly in selected studies. ${ }^{35,40-42,51,53}$ Most studies conducted with lanreotide depot had very few participants withdraw due to adverse effect or lack of efficacy. ${ }^{29}$ Symptoms improved over the course of all studies to varying degrees. Significant improvements have been reported for hyperhidrosis,${ }^{40,42}$ arthralgias, ${ }^{40}$ headache, ${ }^{42}$ and total symptoms. ${ }^{35,51}$ Quality of life, as assessed by a specific questionnaire for acromegaly, was used but demonstrated no improvement in three separate treatment arms from study entry to study end. ${ }^{53}$

\section{Dosage and administration}

Somatuline depot is supplied in $60 \mathrm{mg}, 90 \mathrm{mg}$, and $120 \mathrm{mg}$ doses. The dosing is volume dependent, not concentration dependent, so increasing doses require larger volumes ranging from 0.3 to $0.5 \mathrm{cc}$. The prefilled syringe requires refrigeration and needs to warm to room temperature prior to injection.

The $90 \mathrm{mg}$ dose should be used initially as treatment for acromegaly, given every 4 weeks, for 3 months. According to the prescribing information, the dose should then be titrated according to results of serum GH and IGF-I measurements. Patients who remain uncontrolled, with ongoing clinical symptoms of active acromegaly and serum IGF-I levels elevated above age-matched normal levels, and with a serum $\mathrm{GH}$ level $>2.5 \mathrm{ng} / \mathrm{mL}$, should increase the dose to $120 \mathrm{mg}$ administered every 4 weeks. Based on the current prescribing information, it is recommended that patients who are symptomatically controlled and have a normalized serum IGF-I level with a serum GH level between $1 \mathrm{ng} / \mathrm{mL}$ and $2.5 \mathrm{ng} / \mathrm{mL}$ maintain a dose of $90 \mathrm{mg}$ administered every 4 weeks. Patients and clinicians who prefer to adhere to clinical guidelines for criteria of disease control ${ }^{54}$ may wish to increase the dose to $120 \mathrm{mg}$ every 4 weeks. If the patient's symptoms are controlled, the IGF-I is normalized, and the serum GH level is below $1 \mathrm{ng} / \mathrm{mL}$, then the dose of Somatuline can be reduced to $60 \mathrm{mg}$ every 4 weeks. Recent guidelines regarding the monitoring of patients with acromegaly and the criteria for disease control recommend the use of random, single GH levels in patients receiving somatostatin analogs, ${ }^{55}$ as the GH level obtained after glucose suppression has limited utility. ${ }^{56}$ 
One aspect of approved dosing unique in its approach toward the treatment of acromegaly with somatostatin ana$\operatorname{logs}$ is the ability to utilize an extended dosing interval. In addition to the guidelines outlined above, patients who have achieved disease control with $60 \mathrm{mg}$ or $90 \mathrm{mg}$ administered on a monthly basis can extend the time between injections to every 6-8 weeks with an increase in the dose of lanreotide to $120 \mathrm{mg}$. Studies have been conducted to investigate the efficacy, acceptability, and cost effects of extending the dosing interval beyond monthly. ${ }^{50,53}$ One study, conducted in Germany, enrolled subjects who were previously treated with octreotide LAR doses of $10 \mathrm{mg}, 20 \mathrm{mg}$, and $30 \mathrm{mg}$ monthly and switched them to lanreotide depot $120 \mathrm{mg}$ at intervals of every 56,42 , and 28 days, respectively. ${ }^{53}$ Doses were titrated after three injections based on the serum IGF-I levels. Thirty-seven patients were enrolled and 33 subjects completed the study. The intervals set at the beginning of the study remained the same for most subjects in the study ( $71 \%)$. For 5 subjects the interval needed to be shortened to 28 days and two patients were able to increase their interval during the study period. Sixty percent of subjects entered the study with biochemical disease control by IGF-I and a similar number (63\%) achieved disease control at study end. Subjects who were assigned a more frequent dosing schedule were on higher doses of somatostatin analog medication prior to study entry. These patients may have been more resistant to this class of medications in general and consequently showed the lowest rates of normalization (47\%) at study entry and study end. However, subjects in the longest interval-dosing arm, receiving medication every 56 days, had rates of disease control of $86 \%$ at study entry and study end. Furthermore, mean serum IGF-I levels declined throughout the duration of the study in the 56-day interval arm. Quality of life was measured using the Acromegaly Quality of Life questionnaire and demonstrated no significant change in quality of life across all treatment groups. ${ }^{53}$ These results demonstrate that extended dosing is effective at achieving similar rates of control in subjects who have demonstrated that they are responders to the somatostatin analog class of medications. Investigators judged the injections with lanreotide to be easier than using octreotide LAR and patients preferred receiving their injections less frequently. ${ }^{53}$

Another study approached extended dosing in a different way. ${ }^{50}$ By increasing the interval in nine subjects, and only increasing the dose if disease control was not optimized as measured by combined assessment of GH and IGF-I, they found that some patients using the $60 \mathrm{mg}$ $(n=3)$, the $90 \mathrm{mg}$ dose $(n=2)$, or the $120 \mathrm{mg}$ dose $(n=2)$ could increase the interval between shots to every 6 weeks and maintain disease control over a 24 -week period. This reduced the overall dose and cost of administration. Subjects had previously enrolled in a separate study switching from octreotide LAR to lanreotide ${ }^{35}$ and, after being treated with lanreotide for 24 weeks, they enrolled in this study to evaluate the efficacy of extended dosing. Patients were biochemically controlled at baseline and maintained control with a lower drug requirement in 7/9 subjects. Symptom scores did improve during the study but this difference was not statistically significant.

\section{Place of lanreotide in the treatment of acromegaly}

Lanreotide is one of two commercially available somatostatin analogs used for treatment of acromegaly. Both medications are effective, well tolerated, and are the primary drugs used for medical treatment of acromegaly. Use of one formulation over another depends on many factors including insurance coverage, physician preference, comfort level, and patient preference. Certain aspects of lanreotide, including the drug delivery system, ease of administration, and potential for an extended dosing interval between injections for those who are well controlled on $90 \mathrm{mg}$ or $60 \mathrm{mg}$ doses may have an impact on the choice of drug when treating patients with acromegaly.

\section{Disclosure}

The author has served as an investigator for Ipsen and Tercica (current and prior manufacturers of lanreotide), as a member of advisory boards for Ipsen and Tercica, and is a member of the Lanreotide Curriculum Development Committee.

\section{References}

1. Dekkers OM, Biermasz NR, Pereira AM, Romijn JA, Vandenbroucke JP. Mortality in acromegaly: a metaanalysis. J Clin Endocrinol Metab. 2008;93(1):61-67.

2. Holdaway IM, Bolland MJ, Gamble GD. A meta-analysis of the effect of lowering serum levels of GH and IGF-I on mortality in acromegaly. Eur J Endocrinol. 2008;159(2):89-95.

3. Melmed S. Medical progress: Acromegaly. NEngl J Med. 2006;355(24): 2558-2573.

4. Melmed S. Acromegaly pathogenesis and treatment. J Clin Invest. Nov 2009;119(11):3189-3202.

5. Melmed S, Colao A, Barkan A, et al. Guidelines for acromegaly management: an update. J Clin Endocrinol Metab. 2009;94(5): 1509-1517.

6. Nomikos P, Buchfelder M, Fahlbusch R. The outcome of surgery in 668 patients with acromegaly using current criteria of biochemical "cure." Eur J Endocrinol. 2005;152(3):379-387.

7. Freda PU. Somatostatin analogs in acromegaly. J Clin Endocrinol Metab. 2002;87(7):3013-3018.

8. Alexander L, Appleton D, Hall R, Ross WM, Wilkinson R. Epidemiology of acromegaly in the Newcastle region. Clin Endocrinol (Oxf). 1980; 12(1):71-79. 
9. Fernandez A, Karavitaki N, Wass JA. Prevalence of pituitary adenomas: a community-based, cross-sectional study in Banbury (Oxfordshire, UK). Clin Endocrinol (Oxf). 2010;72(3):377-382.

10. Daly AF, Rixhon M, Adam C, Dempegioti A, Tichomirowa MA, Beckers A. High prevalence of pituitary adenomas: a cross-sectional study in the province of Liege, Belgium. J Clin Endocrinol Metab. 2006;91(12):4769-4775.

11. Nachtigall L, Delgado A, Swearingen B, Lee H, Zerikly R, Klibanski A. Changing patterns in diagnosis and therapy of acromegaly over two decades. J Clin Endocrinol Metab. 2008;93(6):2035-2041.

12. Mestron A, Webb SM, Astorga R, et al. Epidemiology, clinical characteristics, outcome, morbidity and mortality in acromegaly based on the Spanish Acromegaly Registry (Registro Espanol de Acromegalia, REA). Eur J Endocrinol. 2004;151(4):439-446.

13. Holdaway IM, Rajasoorya RC, Gamble GD. Factors influencing mortality in acromegaly. J Clin Endocrinol Metab. 2004;89(2):667-674.

14. Swearingen B, Barker FG 2nd, Katznelson L, et al. Long-term mortality after transsphenoidal surgery and adjunctive therapy for acromegaly J Clin Endocrinol Metab. 1998;83(10):3419-3426.

15. Giustina A, Bronstein MD, Casanueva FF, et al. Current management practices for acromegaly: an international survey. Pituitary. 2011; 14(2):125-133.

16. Trainer PJ, Drake WM, Katznelson L, et al. Treatment of acromegaly with the growth hormone-receptor antagonist pegvisomant. $N$ Engl J Med. 2000;342(16):1171-1177.

17. Buchfelder M, Weigel D, Droste M, et al. Pituitary tumor size in acromegaly during pegvisomant treatment: experience from MR re-evaluations of the German Pegvisomant Observational Study. Eur J Endocrinol. 2009; 161(1):27-35.

18. Cozzi R, Attanasio R, Lodrini S, Lasio G. Cabergoline addition to depot somatostatin analogues in resistant acromegalic patients: efficacy and lack of predictive value of prolactin status. Clinical Endocrinol (Oxf). 2004;61(2):209-215.

19. Neggers SJ, de Herder WW, Janssen JA, Feelders RA, van der Lely AJ. Combined treatment for acromegaly with long-acting somatostatin analogs and pegvisomant: long-term safety for up to 4.5 years (median 2.2 years) of follow-up in 86 patients. Eur J Endocrinol. 2009;160(4): 529-533.

20. Neggers SJ, de Herder WW, Feelders RA, van der Lely AJ. Conversion of daily pegvisomant to weekly pegvisomant combined with long-acting somatostatin analogs, in controlled acromegaly patients. Pituitary. 2011;14(3):253-258

21. Bevan JS. Clinical review: The antitumoral effects of somatostatin analog therapy in acromegaly. J Clin Endocrin Metab. 2005; 90(3):1856-1863.

22. Castinetti F, Nagai M, Morange I, et al. Long-term results of stereotactic radiosurgery in secretory pituitary adenomas. J Clin Endocrin Metab. 2009;94(9):3400-3407.

23. Sherlock M, Ayuk J, Tomlinson JW, et al. Mortality in patients with pituitary disease. Endocr Rev. 2010;31(3):301-342.

24. Ayuk J, Clayton RN, Holder G, Sheppard MC, Stewart PM, Bates AS. Growth hormone and pituitary radiotherapy, but not serum insulin-like growth factor-I concentrations, predict excess mortality in patients with acromegaly. J Clin Endocrinol Metab. 2004;89(4):1613-1617.

25. Ben-Shlomo A, Melmed S. Pituitary somatostatin receptor signaling. Trends Endocrinol Metab. 2010;21(3):123-133.

26. Freda PU, Katznelson L, van der Lely AJ, Reyes CM, Zhao S, Rabinowitz D. Long-acting somatostatin analog therapy of acromegaly: a meta-analysis. J Clin Endocrinol Metab. 2005;90(8):4465-4473.

27. Cendros JM, Peraire C, Troconiz IF, Obach R. Pharmacokinetics and population pharmacodynamic analysis of lanreotide Autogel. Metabolism. 2005;54(10):1276-1281.

28. Foxx-Orenstein A, Camilleri M, Stephens D, Burton D. Effect of a somatostatin analogue on gastric motor and sensory functions in healthy humans. Gut. 2003;52(11):1555-1561.

29. Ipsen Pharmacueticals Somatuline Depot (Lanreotide) injection US prescribing information, 2011.
30. Antonijoan RM, Barbanoj MJ, Cordero JA, et al. Pharmacokinetics of a new Autogel formulation of the somatostatin analogue lanreotide after a single subcutaneous dose in healthy volunteers. J Pharm Pharmacol. 2004;56(4):471-476.

31. Bronstein M, Musolino N, Jallad R, et al. Pharmacokinetic profile of lanreotide Autogel in patients with acromegaly after four deep subcutaneous injections of 60, 90 or $120 \mathrm{mg}$ every 28 days. Clin Endocrinol (Oxf). 2005;63(5):514-519.

32. Troconiz IF, Cendros JM, Peraire C, et al. Population pharmacokinetic analysis of lanreotide Autogel in healthy subjects: evidence for injection interval of up to 2 months. Clin Pharmacokinet. 2009;48(1):51-62.

33. Carmichael JD, Bonert VS. Medical therapy: options and uses. Rev Endocr Metab Disord. 2008;9(1):71-81.

34. Murray RD, Melmed S. A critical analysis of clinically available somatostatin analog formulations for therapy of acromegaly. J Clin Endocrinol Metab. 2008;93(8):2957-2968.

35. Alexopoulou O, Abrams P, Verhelst J, et al. Efficacy and tolerability of lanreotide Autogel therapy in acromegalic patients previously treated with octreotide LAR. E J Endocrinol. 2004;151(3):317-324.

36. Andries M, Glintborg D, Kvistborg A, Hagen C, Andersen M. A 12-month randomized crossover study on the effects of lanreotide Autogel and octreotide long-acting repeatable on GH and IGF-1 in patients with acromegaly. Clin Endocrinol. 2008;68(3):473-480.

37. Ashwell SG, Bevan JS, Edwards OM, et al. The efficacy and safety of lanreotide Autogel in patients with acromegaly previously treated with octreotide LAR. E J Endocrinol. 2004;150(4):473-480.

38. Caron P, Beckers A, Cullen DR, et al. Efficacy of the new long-acting formulation of lanreotide (lanreotide Autogel) in the management of acromegaly. J Clin Endocrin Metab. 2002;87(1):99-104.

39. Caron P, Bex M, Cullen DR, et al. One-year follow-up of patients with acromegaly treated with fixed or titrated doses of lanreotide Autogel. Clin Endocrinol. 2004;60(6):734-740.

40. Colao A, Auriemma RS, Rebora A, et al. Significant tumour shrinkage after 12 months of lanreotide Autogel-120 mg treatment given first-line in acromegaly. Clin Endocrinol(Oxf). 2009;71(2):237-245.

41. Chanson P, Borson-Chazot F, Kuhn JM, Blumberg J, Maisonobe P, Delemer B. Control of IGF-I levels with titrated dosing of lanreotide Autogel over 48 weeks in patients with acromegaly. Clin Endocrinol. 2008;69(2):299-305.

42. Lucas T, Astorga R. Efficacy of lanreotide Autogel administered every 4-8 weeks in patients with acromegaly previously responsive to lanreotide microparticles $30 \mathrm{mg}$ : a phase III trial. Clin Endocrinol (Oxf) 2006;65(3):320-326.

43. Melmed S, Cook D, Schopoh1 J, Goth MI, Lam KS, Marek J. Rapid and sustained reduction of serum growth hormone and insulin-like growth factor- 1 in patients with acromegaly receiving lanreotide Autogel therapy: a randomized, placebo-controlled, multicenter study with a 52 week open extension. Pituitary. 2010;13(1):18-28.

44. Ronchi CL, Boschetti M, Degli Uberti EC, et al. Efficacy of a slowrelease formulation of lanreotide (Autogel) $120 \mathrm{mg}$ in patients with acromegaly previously treated with octreotide long acting release (LAR): an open, multicentre longitudinal study. Clin Endocrinol (Oxf). 2007;67(4):512-519.

45. Toledano Y, Rot L, Greenman Y, et al. Efficacy of long-term lanreotide treatment in patients with acromegaly. Pituitary. 2009;12(4): 285-293.

46. Gutt B, Bidlingmaier M, Kretschmar K, Dieterle C, Steffin B, Schopohl J. Four-year follow-up of acromegalic patients treated with the new long-acting formulation of Lanreotide (Lanreotide Autogel) Exp Clin Endocrinol Diabetes. 2005;113(3):139-144.

47. Caron P, Cogne M, Raingeard I, Bex-Bachellerie V, Kuhn JM. Effectiveness and tolerability of 3-year lanreotide Autogel treatment in patients with acromegaly. Clin Endocrinol. 2006;64(2):209-214.

48. Steffin B, Gutt B, Bidlingmaier M, Dieterle C, Oltmann F, Schopohl J. Effects of the long-acting somatostatin analogue Lanreotide Autogel on glucose tolerance and insulin resistance in acromegaly. Eur J Endocrinol. Jul 2006;155(1):73-78. 
49. Colao A, Marzullo P, Lombardi G. Effect of a six-month treatment with lanreotide on cardiovascular risk factors and arterial intima-media thickness in patients with acromegaly. Eur J Endocrinol. 2002;146(3):303-309.

50. Abrams P, Alexopoulou O, Abs R, Maiter D, Verhelst J. Optimalization and cost management of lanreotide-Autogel therapy in acromegaly. Eur J Endocrinol. 2007;157(5):571-577.

51. Salvatori R, Nachtigall LB, Cook DM, et al. Effectiveness of self- or partner-administration of an extended-release aqueous-gel formulation of lanreotide in lanreotide-naive patients with acromegaly. Pituitary. 2010;13(2):115-122.

52. Bevan JS, Newell-Price J, Wass JA, et al. Home administration of lanreotide Autogel by patients with acromegaly, or their partners, is safe and effective. Clin Endocrinol. 2008;68(3):343-349.
53. Schopohl J, Strasburger CJ, Caird D, et al. Efficacy and acceptability of lanreotide Autogel(R) $120 \mathrm{mg}$ at different dose intervals in patients with acromegaly previously: treated with octreotide LAR. Exp Clin Endocrinol Diabetes. 2011;119(3):156-162.

54. Giustina A, Chanson P, Bronstein MD, et al. A consensus on criteria for cure of acromegaly. J Clin Endocrinol Metab. 2010;95(7): 3141-3148.

55. Giustina A, Chanson P, Bronstein MD, et al. A consensus on criteria for cure of acromegaly. J Clin Endocrinol Metab. 2010;95(7): 3141-3148.

56. Carmichael JD, Bonert VS, Mirocha JM, Melmed S. The utility of oral glucose tolerance testing for diagnosis and assessment of treatment outcomes in 166 patients with acromegaly. J Clin Endocrinol Metab. 2009;94(2):523-527.

\section{Publish your work in this journal}

Patient Preference and Adherence is an international, peer-reviewed, open access journal focusing on the growing importance of patient preference and adherence throughout the therapeutic continuum. Patient satisfaction, acceptability, quality of life, compliance, persistence and their role in developing new therapeutic modalities and compounds to optimize clinical outcomes for existing disease states are major areas of interest. This journal has been accepted for indexing on PubMed Central. The manuscript management system is completely online and includes a very quick and fair peer-review system. Visit http://www.dovepress.com/ testimonials.php to read real quotes from published authors. 